\title{
Giant magneto field effect in up-conversion amplified spontaneous emission via spatially extended states in organic-inorganic hybrid perovskites
}

\author{
Tangyao Shen ${ }^{1, \dagger}$, Jiajun Qin ${ }^{2, \dagger}$, Yujie Bai ${ }^{1, \dagger}$, Jia Zhang ${ }^{3}$, Lei Shi ${ }^{1 *}$, \\ Xiaoyuan $\mathrm{Hou}^{1}$, Jian $\mathrm{Zi}^{1 *}$ and $\mathrm{Bin} \mathrm{Hu}^{3 *}$
}

\begin{abstract}
Up-conversion lasing actions are normally difficult to realize in light-emitting materials due to small multi-photon absorption cross section and fast dephasing of excited states during multi-photon excitation. This paper reports an easily accessible up-conversion amplified spontaneous emission (ASE) in organic-inorganic hybrid perovskites $\left(\mathrm{MAPbBr}_{3}\right)$ films by optically exciting broad gap states with sub-bandgap laser excitation. The broad absorption was optimized by adjusting the grain sizes in the $\mathrm{MAPbBr}_{3}$ films. At low sub-bandgap pumping intensities, directly exciting the gap states leads to 2-photon, 3-photon, and 4-photon up-conversion spontaneous emission, revealing a large optical cross section of multiphoton excitation occurring in such hybrid perovskite films. At moderate pumping intensity $\left(1.19 \mathrm{~mJ} / \mathrm{cm}^{2}\right)$ of $700 \mathrm{~nm}$ laser excitation, a significant spectral narrowing phenomenon was observed with the full width at half maximum (FWHM) decreasing from $18 \mathrm{~nm}$ to $4 \mathrm{~nm}$ at the peak wavelength of $550 \mathrm{~nm}$, simultaneously with a nonlinear increase on spectral peak intensity, showing an up-conversion ASE realized at low threshold pumping fluence. More interestingly, the up-conversion ASE demonstrated a giant magnetic field effect, leading to a magneto-ASE reaching $120 \%$. In contrast, the upconversion photoluminescence $(\mathrm{PL})$ showed a negligible magnetic field effect $(<1 \%)$. This observation provides an evidence to indicate that the light-emitting states responsible for up-conversion ASE are essentially formed as spatially extended states. The angular dependent spectrum results further verify the existence of spatially extended states which are polarized to develop coherent in-phase interaction. Clearly, using broad gap states with spatially extended light-emitting states presents a new approach to develop up-conversion ASE in organic-inorganic hybrid perovskites.
\end{abstract}

Keywords: organic-inorganic hybrid perovskite; orbital order; magneto-ASE; spatially extended states

Shen TY, Qin JJ, Bai YJ, Zhang J, Shi L et al. Giant magneto field effect in up-conversion amplified spontaneous emission via spatially extended states in organic-inorganic hybrid perovskites. Opto-Electron Adv 5, 200051 (2022).

\section{Introduction}

Organic-inorganic hybrid perovskites are known as solution-processing semiconductors with highly efficient light emission covering entire visible spectrum region under down-conversion condition ${ }^{1-5}$. It has been reported that the photoluminescence quantum yield can be as

1Department of Physics, Fudan University, Shanghai 200433, China; ${ }^{2}$ Department of Physics, Chemistry and Biology (IFM), Linköping University, Linköping 58183, Sweden; ${ }^{3}$ Department of Materials Science and Engineering, University of Tennessee, Knoxville, Tennessee 37996 , USA.

tThese authors contributed equally to this work.

*Correspondence: L Shi, E-mail: Ishi@fudan.edu.cn; J Zi, E-mail: jzi@fudan.edu.cn; B Hu, E-mail: bhu@utk.edu

Received: 1 September 2020; Accepted: 30 October 2020; Published online: 28 February 2022

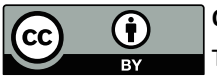

Open Access This article is licensed under a Creative Commons Attribution 4.0 International License.

To view a copy of this license, visit http://creativecommons.org/licenses/by/4.0/.

(C) The Author(s) 2022. Published by Institute of Optics and Electronics, Chinese Academy of Sciences. 
high as $90 \%$ in solid states ${ }^{6-8}$. Recently, such hybrid perovskites have demonstrated lasing actions through ASE at low pumping fluence ${ }^{9-18}$. This development indicates that hybrid perovskites are potential lasing materials with solution processing-tunable properties through down-conversion excitation. Although it is often difficult to realize up-conversion lasing actions in most lightemitting materials due to small multi-photon absorption cross section and dephasing in excited states during multi-photon excitation ${ }^{19-21}$, experimental studies have shown successful up-conversion ASE in both inorganic cesium perovskites and organic-inorganic hybrid $\left(\mathrm{MAPbBr}_{3}\right)$ perovskites ${ }^{22-29}$. This indicates great potential for coherent control of light emitting states through multi-photon excitation in hybrid perovskites. Yet, it still demands further investigations to reveal the underlying mechanisms responsible for up-conversion ASE.

Toward unrevealing the collective emission of up-conversion ASE, magnetic field effect is proposed to be an effective tool. This method has been widely reported in exploring the behavior between light emitting states in organics and halide perovskites ${ }^{30-34}$. Generally, weak coupling of these excited states results in a relatively small magnetic field effects (less than 10\%) $)^{32}$, which hinders its applications in real functional devices. In view of coherent emission in ASE region, much stronger coupling of excited states for collective correlations is expected, giving rise to a great potential to facilitate magnetic field effects.

In this work, we prepare the perovskite $\left(\mathrm{MAPbBr}_{3}\right)$ films with large grains in scale of several micrometers through our previous reported method m $^{35,36}$ to investigate the possibilities of using gap states to realize up-conversion ASE. By absorption measurements, high broadband absorption is observed in the sub-bandgap energy region, indicating the formation of gap states in spectral region from $600 \mathrm{~nm}$ to $1300 \mathrm{~nm}$. These gap states give rise to a large cross section of multiphoton absorption, resulting in strong up-conversion PL under low pumping intensities. More interestingly, the up-conversion ASE shows a significant increasement (over 120\%) upon applying the magnetic field of $1.2 \mathrm{~T}$, generating giant magnetic field effects. The observed giant magnetic field effects provide an evidence to show that an orbital order is formed in spatially extended states by exciting broad gap states. Essentially, with orbital order the spatially extended states develop a cooperative interaction towards generating an ASE in hybrid halide perovskites prepared with large grains through fast crystallization.

\section{Materials and methods}

\section{Preparation of perovskite $\left(\mathrm{MAPbBr}_{3}\right)$ films}

The perovskite films were spin-coated with the thickness of 1 micrometer onto the ITO/PEDOT:PSS substrates at $\mathrm{N}_{2}$ atmosphere. Essentially, the perovskite films were prepared with one-step spin-coating method by using the precursor solution $\left(1.5 \mathrm{mmol} \mathrm{PbBr}_{2}\right.$ and $1.58 \mathrm{mmol}$ $\mathrm{MABr}$ in $1 \mathrm{~mL}$ DMF solution) at the spinning rate of $3000 \mathrm{rpm}$ for 1 minute, followed by annealing at $60{ }^{\circ} \mathrm{C}$ for 30 minutes. The grain size can be largely changed by partially replacing DMF with DMSO. Prior to the preparation of perovskite films, the PEDOT:PSS films were spin-coated on glass substrates with the thickness of 40 $\mathrm{nm}$, followed with the thermal annealing at $150{ }^{\circ} \mathrm{C}$ for 0.5 h. With the glass/PEDOT substrates, high-quality perovskite films can be formed through spin coating.

\section{Measurement of absorption spectrum}

A thin-film transmittance and reflectance spectrometer (RT1736, Ideaoptics, China) was used to measure the absorption of the sample with an integrating sphere (R7, Ideaoptics, China) to eliminate the influence of scattering, equipped with a visible spectrometer (PG2000-pro, Ideaoptics, China) and a NIR spectrometer (NIR1700, Ideaoptics, China). A halogen lamp and a deuterium lamp were used as visible and infrared light source. The transmittance and reflectance of the sample and the substrate were measured, and then the absorption can be derived by the formula $A=1-T-R$. The absorption of substrate was deducted from the whole absorption of the sample to derive the net absorption of the perovskite film.

\section{Measurement of photoluminescence spectrum}

All pumping wavelengths in the experiment were selected from a Coherent Legend regenerative amplifier (150 fs, $1 \mathrm{kHz}, 800 \mathrm{~nm}$ ) which is seeded by a Coherent Mira 900 oscillator (100 fs, $80 \mathrm{MHz}$ ). The Mira is seeded by a Coherent Verdi Laser Generator (continuous wave, 532 $\mathrm{nm}$ ). A Coherent Opera Solo was used to convert the 800 $\mathrm{nm}$ pulse laser to wavelength suitable for the experiment, without changing other parameters. The excitation beam was focused on the sample with the diameter of about 100 micrometers. The output signals were sent into the spectrometer (Horiba, iHR320)) and detected by a 
charge coupled device (Horiba, Synapse).

\section{Measurement of angular dependence}

An R1 Angular-Resolved System (R1, Ideaoptics, China) equipped with a visible spectrometer (PG2000, Ideaoptics, China) was used to measure the angular dependence of the emission from the perovskite $\left(\mathrm{MAPbBr}_{3}\right)$ film. The sample was fixed vertically, and a photodetector was fixed on a mechanical arm which can rotate around a horizontal axis in the film plane. The excitation beam focused perpendicularly on the sample intersecting with the axis by a lens. By rotating the mechanical arm to different position, photoluminescence in different emitting angle can be measured.

\section{Measurement of magnetic field effects}

An electromagnet was used to generate a constant magnetic field of $1.2 \mathrm{~T}$ paralleled to the perovskite $\left(\mathrm{MAPbBr}_{3}\right)$ film. The constant magnetic field was switched on and off through electrical current during upconversion ASE measurement to generate magneto-ASE phenomena. Excitation beam was focused on the sample perpendicularly by a lens. The light emission intensity was recorded once per second with 10 average times by a
THORLABS PDA36A2 silicon photodetector, with several filters before the detector to eliminate excitation beam. The measurement was started with the electromagnet turned off. After 30 points were collected, the measurement was paused until the electromagnet was switched on and the constant current was kept to generate the magnetic field of $1.2 \mathrm{~T}$. The electromagnet was then switched off to complete the measurement cycle.

\section{Results and discussion}

The scanning electron microscopy (SEM) image in Fig. (a) shows that the perovskite $\left(\mathrm{MAPbBr}_{3}\right)$ film is formed with large cubic grains in the order of micrometers. The inset shows the image taken by optical microscopy. Figures S1 and Fig. 1(b) displays the optical absorption as well as PL and excitation spectrum. The film demonstrates a relatively strong absorption (about $50 \%$ ) from $400 \mathrm{~nm}$ to $540 \mathrm{~nm}$. The absorption begins to drop sharply to about $30 \%$ at $540 \mathrm{~nm}$, indicating an optical bandgap of $2.23 \mathrm{eV}(557 \mathrm{~nm})$. Moreover, the absorption shows an appreciable intensity below the bandgap decreasing slightly from about $20 \%$ to $10 \%$ with the wavelength changing from $550 \mathrm{~nm}$ to $1200 \mathrm{~nm}$. At infrared region between $1200 \mathrm{~nm}$ to $1600 \mathrm{~nm}$, the
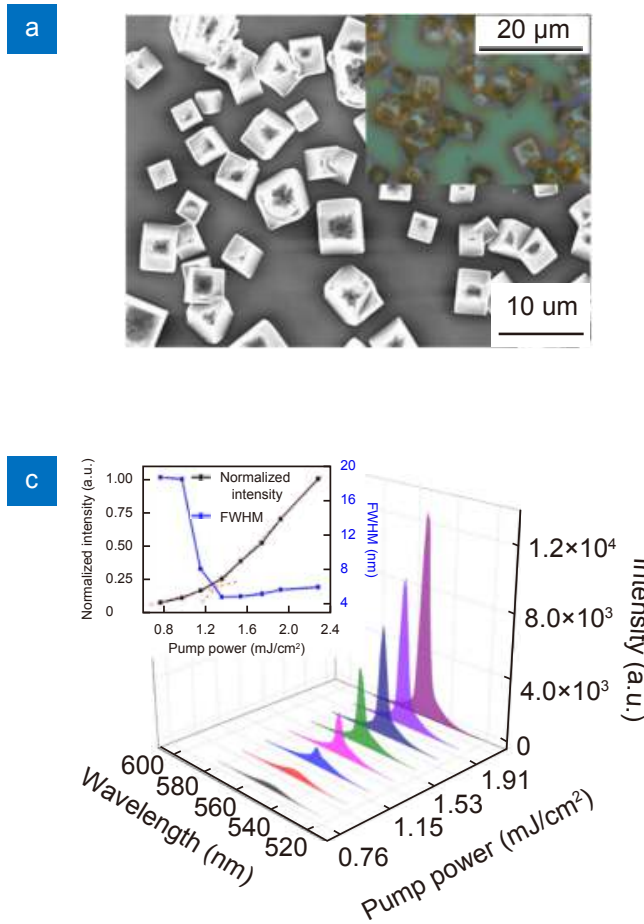

b

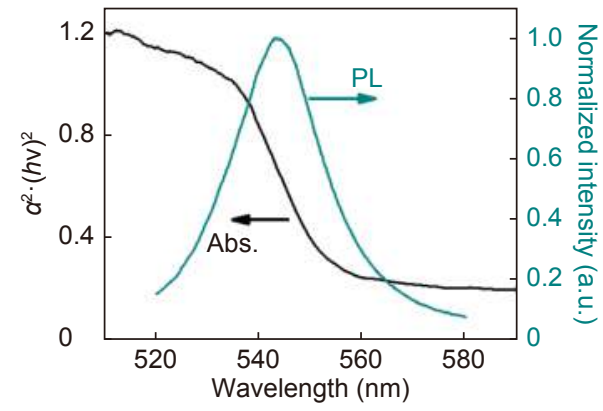

d

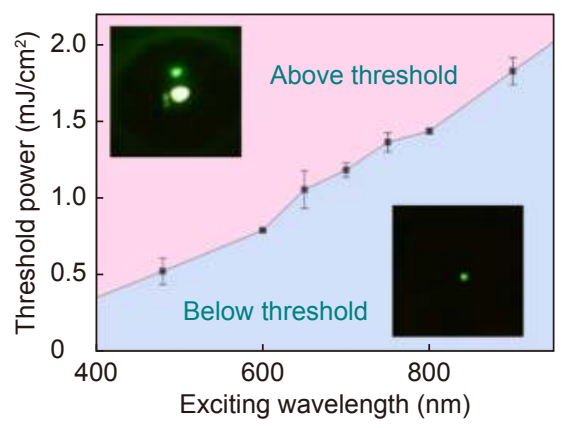

Fig. 1 | Morphological and spectral characterizations. (a) SEM image to show large grains with micrometer sizes. (b) Optical absorption spectrum (inset: band edge with PL). (c) Up-conversion ASE under $700 \mathrm{~nm}$ excitation wavelength (threshold pumping fluence: $1.19 \mathrm{~mJ} / \mathrm{cm}^{2}$ ). Inset: spectral narrowing with emission intensity simultaneously increasing phenomena. (d) Threshold dependence on excitation wavelength. Inset: luminescence above (the upper left) and below (the bottom right) threshold. 
perovskite film still shows an acceptable absorption (about $10 \%$ ), corresponding to $1.04 \mathrm{eV}$ to $0.78 \mathrm{eV}$ (much smaller than the bandgap of $2.23 \mathrm{eV}$ ). Clearly, the perovskite $\left(\mathrm{MAPbBr}_{3}\right)$ films with the large cubic grains of micrometers are formed with broad gap states. Here, we suggest that the broad gap states are formed through structural deformation on large grains through fast crystallization. Theoretically, increasing the grain sizes can essentially lower the potential barriers to form structural deformation during crystallization. As shown in Fig. 1 (c), at low pumping fluence $\left(<0.76 \mathrm{~mJ} / \mathrm{cm}^{2}\right.$ at $\left.700 \mathrm{~nm}\right)$, a broad PL was observed with the FWHM of $\sim 18.5 \mathrm{~nm}$ and the peak position of $546.6 \mathrm{~nm}$. As the pumping fluence was increased from $0.76 \mathrm{~mJ} / \mathrm{cm}^{2}$ to $2.27 \mathrm{~mJ} / \mathrm{cm}^{2}$, a spectral narrowing phenomenon was observed with the FWHM decreased from $18.6 \mathrm{~nm}$ to $4.82 \mathrm{~nm}$, resulting in a sharp peak at $550 \mathrm{~nm}$. Simultaneously, the PL intensity is nonlinearly increasing with pumping fluence, presenting an efficient up-conversion ASE above the pumping threshold of $1.19 \mathrm{~mJ} / \mathrm{cm}^{2}$ by directly exciting the gap states. Similar up-conversion ASE with spectral peak at $550 \mathrm{~nm}$ can also be observed when the perovskite $\left(\mathrm{MAPbBr}_{3}\right)$ films were excited by different laser wavelengths from $480 \mathrm{~nm}$ to $900 \mathrm{~nm}(2.59 \mathrm{eV}$ to 1.38 $\mathrm{eV}$ ), shown as a spectral narrowing phenomenon with nonlinearly increasing intensity above threshold pumping fluences. However, the threshold pumping fluence increases with shifting sub-bandgap excitation to longer wavelength, as shown in Fig. 1(d) (Details for fluence dependent emission spectra can be found in Fig. S2). Clearly, directly exciting broad gap states presents a new approach to develop up-conversion ASE in hybrid perovskite $\left(\mathrm{MAPbBr}_{3}\right)$ films. To note that the well-defined perovskite cubic grains are relatively good cavities, which can lead to lasing action with the FWHM below $1 \mathrm{~nm}^{37,38}$. In our perovskite film, the poor quality of cubic shape fails to form cavities for lasing. Moreover, the concentrated microcrystals behave more like a polycrystal film, which shows ASE with FWHM of around $4 \mathrm{~nm}$ at high fluences. This light amplification indicates that our film is a good gain medium even under below-bandgap excitation.

The multi-photon absorption process can be categorized into two types: one-step process and multi-step process. For the first type, the absorption process is realized on condition that the multiple photons are coherent and have wavefunctions overlap with the initial and final state of the material. Thus, switching the excitation source from coherent one to non-coherent one will cause largely reduced absorption cross section. This process is irrelevant to the gap states. In contrast, the multi-step process in the multi-photon absorption is influenced by the gap states. The electron in valance band is firstly excited into the gap when one below-bandgap photon is absorbed, followed by latter step(s) to excite the electron into conduction band. In this situation, the gap states play an important role in determining the absorption cross section. Specifically, the magnitude is irrelevant to the excitation source, i.e. coherent and non-coherent excitation sources have the same absorption cross section. In our experiment, we compared the ASE spectra at identical pump fluence excited by coherent and noncoherent light sources (Fig. S3), which shows no difference. It verifies that the multi-photon absorption process is a multi-step process in our perovskite films with large perovskite grains. Therefore, the broad gap states revealed by absorption data suggest high multi-photon absorption cross section. Besides, we have compared the gapstate absorptions of small grains and the optimized large grains. It shows that the absorption of small crystals is much lower, indicating lower multi-photon absorption. Moreover, no ASE is observed even when the fluence (Fig. S4) is very high.

To further explore multi-photon absorption through broad gap states, the infrared excitation wavelength was largely tuned from $900 \mathrm{~nm}$ to $1200 \mathrm{~nm}$ and $1500 \mathrm{~nm}$ to detect up-conversion light emission. The power-law is effective to identify the types of radiative recombination only when we consider the PL intensities at initial time, i.e. $\mathrm{PL}_{0}$ in transient $\mathrm{PL}$ measurement ${ }^{39}$. In this case, the logarithmic slope of 1 or 2 corresponds to excitonic emission $\left(P L_{0} \propto n_{0}\right)$ or bimolecular emission $\left(P L_{0} \propto n_{0}^{2}\right)$ for down-conversion condition. Specifically, the integrated $\mathrm{PL}$ intensity $\left(I_{\mathrm{PL}}\right)$ exciting with femtosecond pulse laser is proportional to the integration of recombination, i.e. $I_{\mathrm{PL}} \propto \int_{0}^{+\infty} n \mathrm{~d} t$ for excitonic emission or $I_{\mathrm{PL}} \propto \int_{0}^{+\infty} n^{2} \mathrm{~d} t$ for bimolecular emission. When the carrier density increases to high value, the bimolecular recombination or Auger recombination will dominate, leading to the decrease of logarithmic slop ${ }^{40,41}$. In Fig. S5, the logarithmic slope at excitation of $400 \mathrm{~nm}$ (onephoton absorption) is 1.31 , indicating the dominance of high-order recombination(s) (bimolecular and/or Auger). When the excitation wavelength is $900 \mathrm{~nm}$, the emission spectra for spontaneous emission are the same 

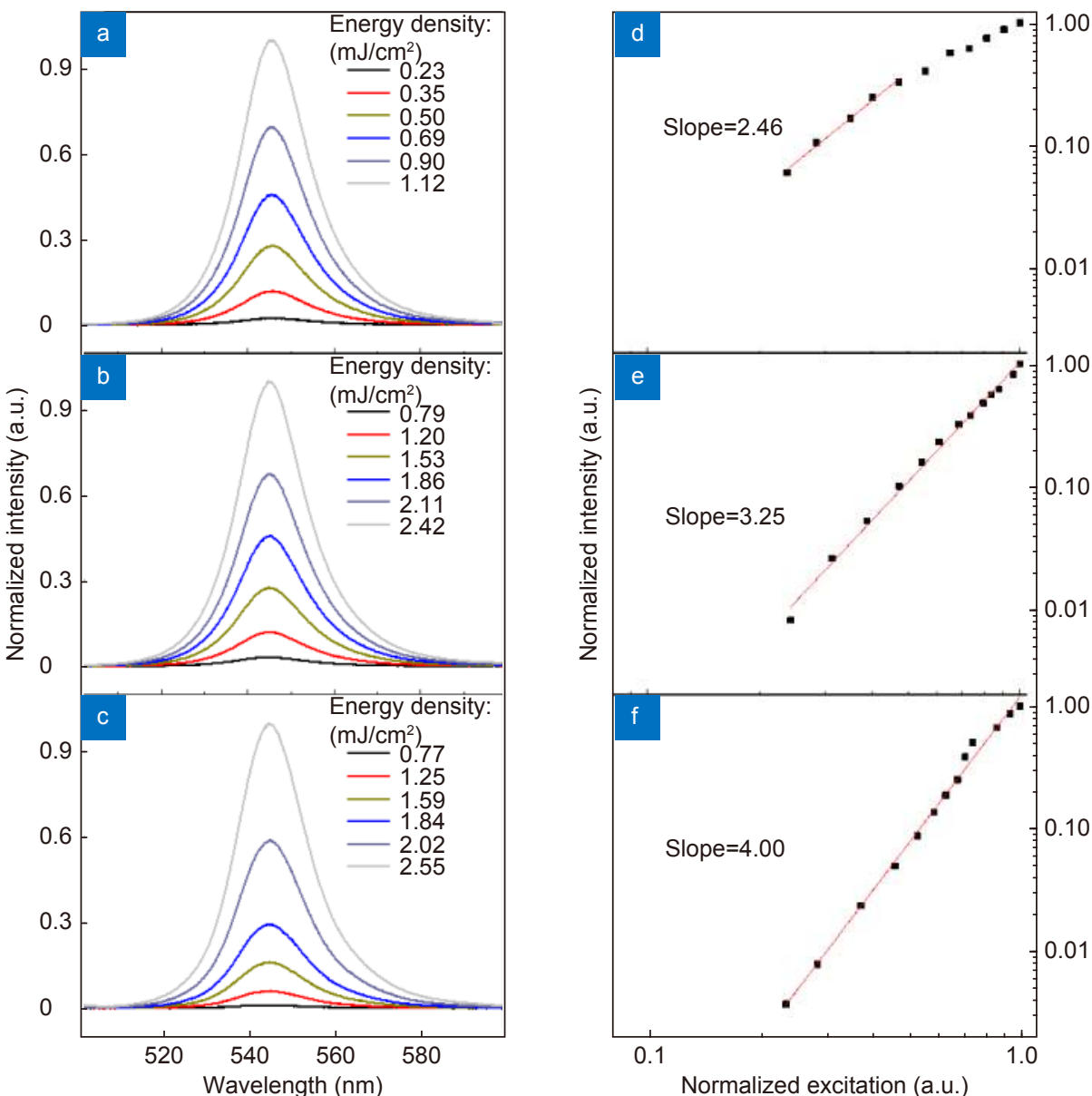

Fig. 2 | Multi-photon up-conversion PL and intensity dependence. (a, d) 2-photon process under $900 \mathrm{~nm}$ excitation wavelength. (b),(e) 3photon process under $1200 \mathrm{~nm}$ excitation wavelength. (c, f) 4-photon process under $1500 \mathrm{~nm}$ excitation wavelength.

(Fig. 2(a)) while the logarithmic slope increases to 2.46 ((Fig. 2(d)). This value is close to the twice of the slope excited by $400 \mathrm{~nm}$, implying that $900 \mathrm{~nm}$ excitation leads to two-photon absorption process in our perovskite film. When the excitation wavelength is $1200 \mathrm{~nm}$ or $1500 \mathrm{~nm}$ (Fig. 2(b, c, e, f)), the logarithmic slope tends to be closer to 3 or 4 . We should note that, the absorption coefficient decreases significantly with the increasing excitation wavelength, resulting in very low carrier density. Although bimolecular recombination is the main radiative recombination in $3 \mathrm{D}$ perovskite film, the "excitonic-like" emission $\left(I_{\mathrm{PL}} \propto n_{0}\right)$ appears at very low carrier density $n$, which is attributed to a pseudo-first-order process (e.g. $\left.n_{\text {injected }}<<n_{\text {intrinsic }}\right)^{39}$. Therefore, the logarithmic slope of 3 and 4 correspond to the three-photon and four-photon process. This observation indicates that broad gaps states possess strong multi-photon absorption characteristics towards developing multi-photon up-conversion $\mathrm{PL}$ in hybrid halide perovskites. We should note that multiphoton up-conversion probability is directly related to the correlation between gap states during multi-photon absorption. Here, we can see that the large grains with the sizes in micrometers can demonstrate the high probabilities to enable multi-photon up-conversion PL in hybrid perovskite films. This indicates that the gap states possess spatially extended wavefunctions to increase multi-photon absorption probability in large grains.

As up-conversion ASE occurred in the perovskite $\left(\mathrm{MAPbBr}_{3}\right)$ films, the light-emitting states become strongly coupled with each other, which is supposed to provide large response to magnetic field. Therefore, we explore the magnetic field effects of light-emitting states responsible for up-conversion ASE. As reported, magnetic field effects can be observed when an external magnetic field is able to disturb the conversion between different states ${ }^{42-46}$. Here, Fig. 3(a) shows the magneto-ASE, where the ASE intensity is plotted with and without the magnetic field of $1.2 \mathrm{~T}$ under two-photon excitation (800 $\mathrm{nm})$. Interestingly, applying a static magnetic field of 1.2 $\mathrm{T}$ can significantly increase the up-conversion ASE 

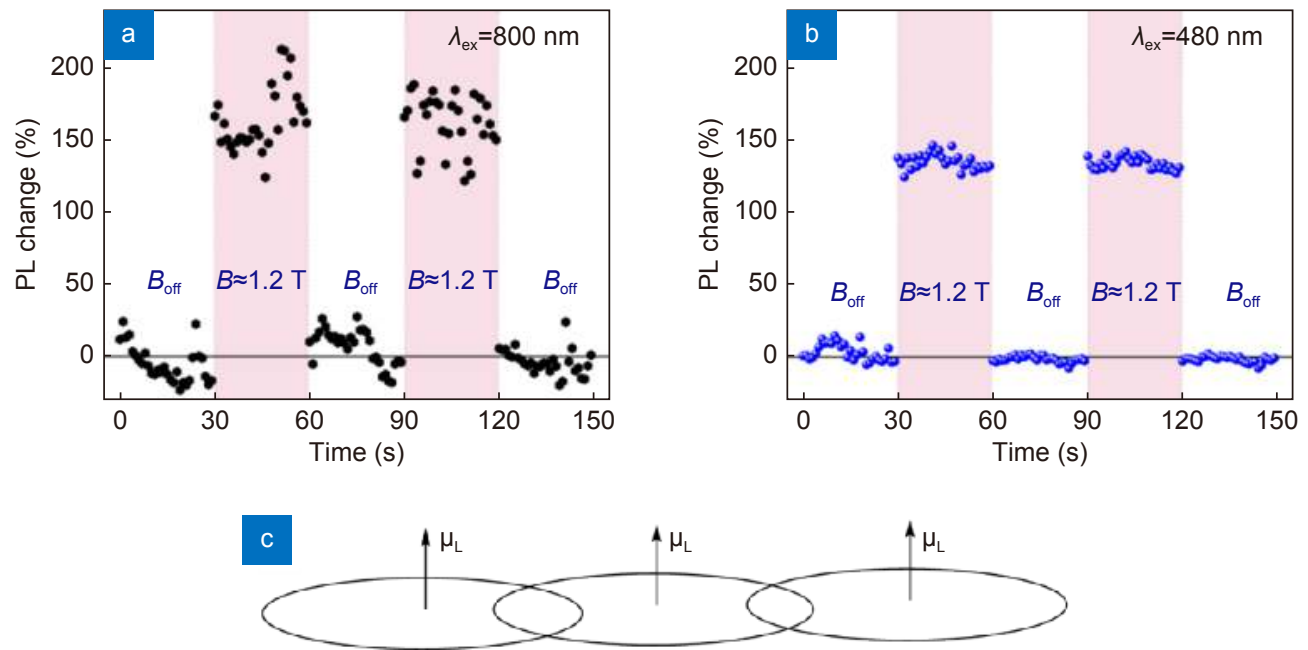

Fig. 3 | ASE measured with and without magnetic field in both up- and down-conversion regions. Magnetic field: 1.2 T. (a) Up-conversion ASE under $800 \mathrm{~nm}$ excitation wavelength. (b) Down-conversion ASE under $480 \mathrm{~nm}$ excitation wavelength. (c) Orbital order: orbitals are aligned through magnetic dipoles to form cooperative light-emitting states towards developing an ASE.

intensity by about $150 \%$ at the pumping fluence $(>1.91$ $\mathrm{mJ} / \mathrm{cm}^{2}$ ) above the threshold intensity, leading to a giant magneto-ASE in up-conversion regime. It should be pointed out that the value (150\%) in ASE region is much higher than the commonly reported value (less than 1\%) in spontaneous emission for hybrid perovskite ${ }^{32}$. This observation provides a strong evidence that spatially extended states are essentially formed when directly exciting gap states with the two-photon pumping fluence above ASE threshold.

We should note that the spatially extended states are the states with delocalized wavefunctions, which are supposed to be realized through orbital order interaction. When an orbital order is established between spatially extended states, the light-emitting states can develop a cooperative interaction, providing the necessary condition to realize an ASE. Dynamically, this requires that the orbital order needs to be at least partially conserved during ASE. More importantly, when an external magnetic field suppresses the relaxation of orbital order to enhance the cooperative interaction between light-emitting states, ASE intensity can be largely increased, leading to magnetic field effects with the magnitude larger than $100 \%$. Essentially, the orbital order leads to cooperative light-emitting states towards realizing up-conversion ASE. To verify the spatially extended states with cooperative order, magnetic field effects in down-conversion ASE were measured. Figure 3(b) shows the magneto-ASE in down-conversion condition under the pulse excitation of $480 \mathrm{~nm}$ with the pumping energy of $>1.27$ $\mathrm{mJ} / \mathrm{cm}^{2}$. Obviously, applying the static magnetic field of
1.2 $\mathrm{T}$ can also significantly increase the down-conversion ASE intensity, giving rise to a giant magneto-ASE of $120 \%$ in down conversion regime when the $480 \mathrm{~nm}$ pumping fluence exceeding the ASE threshold intensity, while the down-conversion PL shows negligible response to the static magnetic field $(<1 \%)$ below ASE threshold. Clearly, the giant magneto-ASE provides the direct evidence that the orbital order is established between spatially extended states are formed, providing the necessary condition to generate cooperative interaction between light-emitting states towards developing an ASE, when the pumping intensity exceeds the threshold fluence in hybrid perovskites, as schematically shown in Fig. 3(c). The orbital order refers to the alignment of orbital-magnetic dipoles in order to develop coherent light-emitting states towards ASE. The giant magnetoASE implies that a magnetic field can further increase the alignment of orbital-magnetic dipoles, significantly increasing ASE. Therefore, the orbital order plays an important role to develop cooperative light-emitting states towards ASE in hybrid perovskites. For higher-quality crystals like perfect perovskite cuboids ${ }^{47}$, the recombination loss would be reduced, which in principle leads to lower lasing threshold. In this situation, charged defects would be reduced, which minimizes the screening effect on light emitting states toward increased delocalization. Therefore, the orbital order interaction between the excited states is supposed to be enhanced. As a result, higher magneto-ASE signal is expected.

To further understand the characteristics of spatially extended states by directly exciting broad gap states, we 
investigate the spatial distribution of up-conversion ASE by monitoring the angle-resolved emission intensity. The angle-resolved experimental system was used to measure PL intensity at variable angles ranging from $0^{\circ}$ to $90^{\circ}$ relative to the film plane, with the excitation beam (700 $\mathrm{nm}$ ) perpendicularly focused on the film plane, as depicted in Fig. 4(a). Figure 4(b) demonstrated that at pumping fluence $\left(0.68 \mathrm{~mJ} / \mathrm{cm}^{2}\right)$ below the threshold intensity, the up-conversion PL (fluorescence) shows a very limited elliptical spatial distribution. Interestingly, when the pumping fluence is increased to $1.70 \mathrm{~mJ} / \mathrm{cm}^{2}$ (above the threshold), the up-conversion ASE demonstrates a clear asymmetric spatial distribution with the emission intensity sharply decreased at $90^{\circ}$ direction. According to the more elliptical spatial distribution of ASE intensity than spontaneous emission in Fig. 4(b), the transition dipoles responsible for ASE tend to be vertically aligned ${ }^{35}$. In order to realize collective emission, linearly polarized ASE emission with the direction parallel to the transition dipoles is suggested. Thus, the output should be linearly polarized perpendicularly to the film plane. This implies that the spatially extended states are orientated towards the direction perpendicular to film plane. Similar results can be observed when we change the excitation wavelength to $480 \mathrm{~nm}$ (down-conversion) and $800 \mathrm{~nm}$ (up-conversion) in Fig. S6, verifying that the collective emission is universal for ASE in our perovskite film. Es-
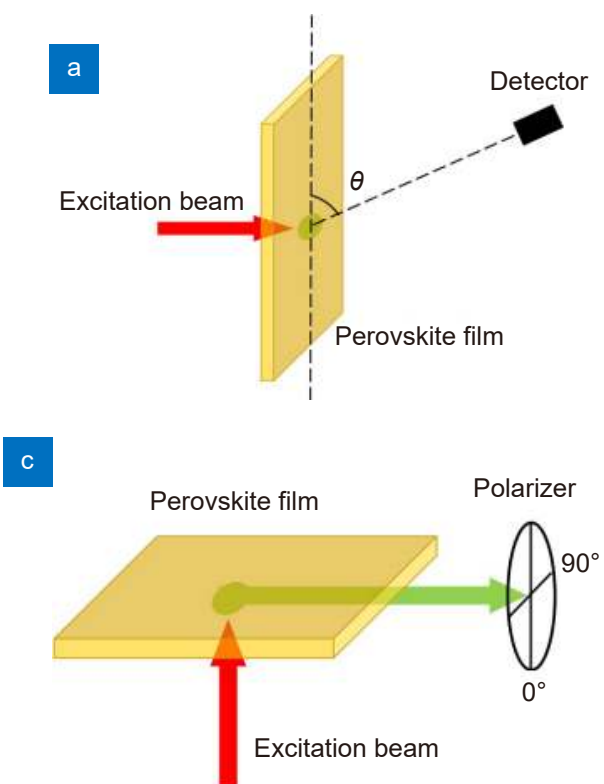

sentially, this collective emission is further confirmed by measuring the polarization of ASE output as illustrated in Fig. 4(c). The result shown in Fig. 4(d) demonstrates that, when up-conversion ASE occurred, the output is linearly polarized with the direction perpendicular to the film plane, where the degree of polarization (DOP) reaches $11.1 \%$. For other excitation wavelengths $(480 \mathrm{~nm}$ and $800 \mathrm{~nm}$ in Fig. S7), polarized ASE emission can always be characterized. This result verifies that spatially extended states with cooperative order are indeed orientated, providing the necessary condition to develop coherent interaction towards developing an ASE.

\section{Conclusion}

In summary, up-conversion ASE is realized by directly exciting the broad gap states in hybrid perovskite $\left(\mathrm{MAPbBr}_{3}\right)$ films prepared with large cubic grains in the order of micrometers. The 2-photon, 3-photon, and 4photon PL indicates that the broad gap states possess strong multi-photon absorption characteristics, providing the necessary condition to generate up-conversion light emission. By directly exciting broad gap states with NIR pumping fluence exceeding threshold intensity, a spectral narrowing phenomenon in up-conversion light emission was observed, showing as an up-conversion ASE in perovskite $\left(\mathrm{MAPbBr}_{3}\right)$ films prepared with large grains. Importantly, a static magnetic field of $1.2 \mathrm{~T}$ can
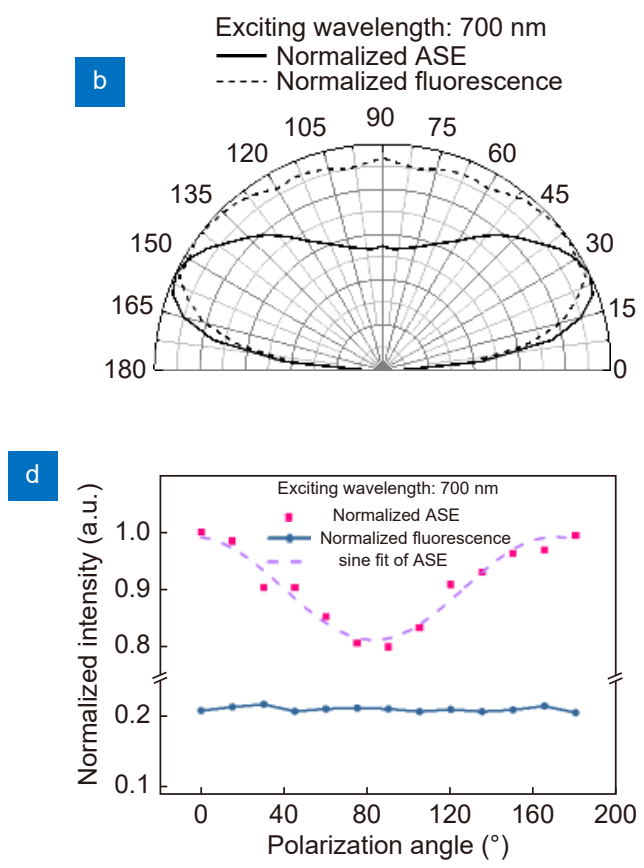

Fig. 4 | Angle and polarization dependences for ASE and PL. (a) Setup for angle dependence measurement. (b) Spatial distributions of ASE and PL outputs. (c) Setup for polarization measurement. (d) Polarizations of ASE and PL outputs. 
significantly increase the ASE intensity by more than $150 \%$, leading to giant magnetic field effects in up-conversion ASE regime. The observed giant magnetic field effects provide an evidence to show that the orbital order is established between spatially extended states in the hybrid perovskite $\left(\mathrm{MAPbBr}_{3}\right)$ films. Essentially, the orbital order between spatially extended states provides the necessary condition to generate a cooperative interaction between light-emitting states towards realizing upconversion ASE. This is verified by similar phenomenon in down-conversion ASE: giant magnetic field effects (120\%) are also observed in down-conversion ASE. Clearly, with broad gap states, establishing the orbital order between spatially extended states presents a new mechanism to realize up-conversion ASE in hybrid halide perovskites. This creates new opportunities to use hybrid halide perovskites in infrared sensing, biological detection, and lasing technologies. Moreover, the giant magnetic field effects observed in ASE regime provides the promise to utilize hybrid halide perovskites in spintronics and biological magnetic probing.

\section{References}

1. Hao F, Stoumpos CC, Chang RPH, Kanatzidis MG. Anomalous band gap behavior in mixed $\mathrm{Sn}$ and $\mathrm{Pb}$ perovskites enables broadening of absorption spectrum in solar cells. J Am Chem Soc 136, 8094-8099 (2014).

2. De Wolf S, Holovsky J, Moon SJ, Löper P, Niesen B et al. Organometallic halide perovskites: sharp optical absorption edge and its relation to photovoltaic performance. J Phys Chem Lett 5, 1035-1039 (2014).

3. Ahmadi M, Wu T, Hu B. A review on organic-inorganic halide perovskite photodetectors: device engineering and fundamental physics. Adv Mater 29, 1605242 (2017).

4. Kazim S, Nazeeruddin MK, Grätzel M, Ahmad S. Perovskite as light harvester: a game changer in photovoltaics. Angew Chem Int Ed 53, 2812-2824 (2014).

5. Ball JM, Stranks SD, Hörantner MT, Hüttner S, Zhang W et al. Optical properties and limiting photocurrent of thin-film perovskite solar cells. Energy Environ Sci 8, 602-609 (2015).

6. Quan LN, Quintero-Bermudez R, Voznyy O, Walters G, Jain A et al. Highly emissive green perovskite nanocrystals in a solid state crystalline matrix. Adv Mater 29, 1605945 (2017).

7. Li Z, Kong L, Huang S, Li L. Highly luminescent and ultrastable $\mathrm{CsPbBr}_{3}$ perovskite quantum dots incorporated into a silica/alumina monolith. Angew Chem Int Ed 56, 8134-8138 (2017).

8. Veldhuis SA, Boix PP, Yantara N, Li MJ, Sum TC et al. Perovskite materials for light-emitting diodes and lasers. Adv Mater 28, 6804-6834 (2016).

9. Xing GC, Mathews N, Lim SS, Yantara N, Liu XF et al. Lowtemperature solution-processed wavelength-tunable perovskites for lasing. Nat Mater 13, 476-480 (2014).

10. Jia YF, Kerner RA, Grede AJ, Rand BP, Giebink NC. Continuous-wave lasing in an organic-inorganic lead halide perovskite semiconductor. Nat Photonics 11, 784-788 (2017).

11. Jia YF, Kerner RA, Grede AJ, Brigeman AN, Rand BP et al. Diode-pumped organo-lead halide perovskite lasing in a metalclad distributed feedback resonator. Nano Lett 16, 4624-4629 (2016).

12. Dhanker R, Brigeman AN, Larsen AV, Stewart RJ, Asbury JB et al. Random lasing in organo-lead halide perovskite microcrystal networks. App/ Phys Lett 105, 151112 (2014).

13. Arora N, Dar MI, Hezam M, Tress W, Jacopin G et al. Photovoltaic and amplified spontaneous emission studies of high-quality formamidinium lead bromide perovskite films. Adv Funct Mater 26, 2846-2854 (2016).

14. Wang KY, Li G, Wang S, Liu S, Sun WZ et al. Dark-field sensors based on organometallic halide perovskite microlasers. Adv Mater 30, 1801481 (2018).

15. Yakunin S, Protesescu L, Krieg F, Bodnarchuk MI, Nedelcu G et al. Low-threshold amplified spontaneous emission and lasing from colloidal nanocrystals of caesium lead halide perovskites. Nat Commun 6, 8056 (2015).

16. Evans TJS, Schlaus A, Fu YP, Zhong XJ, Atallah TL et al. Continuous-wave lasing in cesium lead bromide perovskite nanowires. Adv Opt Mater 6, 1700982 (2018).

17. Fu YP, Zhu HM, Schrader AW, Liang D, Ding Q et al. Nanowire lasers of formamidinium lead halide perovskites and their stabilized alloys with improved stability. Nano Lett 16, 1000-1008 (2016).

18. Zhu HM, Fu YP, Meng F, Wu XX, Gong ZZ et al. Lead halide perovskite nanowire lasers with low lasing thresholds and high quality factors. Nat Mater 14, 636-642 (2015).

19. He GS, Yong KT, Zheng QD, Sahoo Y, Baev A et al. Multiphoton excitation properties of CdSe quantum dots solutions and optical limiting behavior in infrared range. Opt Express 15, 12818-12833 (2007).

20. Tian YP, Li L, Zhang JZ, Yang JX, Zhou HP et al. Investigations and facile synthesis of a series of novel multi-functional twophoton absorption materials. J Mater Chem 17, 3646-3654 (2007).

21. Oliveira SL, Corrêa DS, Misoguti L, Constantino CJL, Aroca RF et al. Perylene derivatives with large two-photon-absorption cross-sections for application in optical limiting and upconversion lasing. Adv Mater 17, 1890-1893 (2005).

22. Xu YQ, Chen Q, Zhang CF, Wang R, Wu H et al. Two-photonpumped perovskite semiconductor nanocrystal lasers. J Am Chem Soc 138, 3761-3768 (2016).

23. Nagamine G, Rocha JO, Bonato LG, Nogueira AF, Zaharieva Z et al. Two-photon absorption and two-photon-induced gain in perovskite quantum dots. J Phys Chem Lett 9, 3478-3484 (2018).

24. Pan J, Sarmah SP, Murali B, Dursun I, Peng W et al. Air-stable surface-passivated perovskite quantum dots for ultra-robust, single- and two-photon-induced amplified spontaneous emission. J Phys Chem Lett 6, 5027-5033 (2015).

25. Li CL, Zang ZG, Han C, Hu ZP, Tang XS et al. Highly compact $\mathrm{CsPbBr}_{3}$ perovskite thin films decorated by $\mathrm{ZnO}$ nanoparticles for enhanced random lasing. Nano Energy 40, 195-202 (2017).

26. Zheng Z, Wang XX, Shen YW, Luo ZY, Li LG et al. Space-confined synthesis of $2 \mathrm{D}$ all-inorganic $\mathrm{CsPbl}_{3}$ perovskite nanosheets for multiphoton-pumped lasing. Adv Opt Mater 6, 1800879 (2018).

27. Liao $Q$, Jin $X, F u$ HB. Tunable halide perovskites for 
miniaturized solid-state laser applications. Adv Opt Mater 7, 1900099 (2019).

28. Wang Y, Li XM, Zhao X, Xiao L, Zeng HB et al. Nonlinear absorption and low-threshold multiphoton pumped stimulated emission from all-inorganic perovskite nanocrystals. Nano Lett 16, 448-453 (2016).

29. Yang DC, Xie C, Sun JH, Zhu H, Xu XH et al. Amplified spontaneous emission from organic-inorganic hybrid lead iodide perovskite single crystals under direct multiphoton excitation. Adv Opt Mater 4, 1053-1059 (2016).

30. He L, Li MX, Urbas A, Hu B. Optically tunable magneto-capacitance phenomenon in organic semiconducting materials developed by electrical polarization of intermolecular charge-transfer States. Adv Mater 26, 3956-3961 (2014).

31. Hu B, Yan L, Shao M. Magnetic-field effects in organic semiconducting materials and devices. Adv Mater 21, 1500-1516 (2009).

32. Xu HX, Wang MS, Yu ZG, Wang K, Hu B. Magnetic field effects on excited states, charge transport, and electrical polarization in organic semiconductors in spin and orbital regimes. Adv Phys 68, 49-121 (2019).

33. Zhang J, Hu B. Revealing photoinduced bulk polarization and spin-orbit coupling effects in high-efficiency 2D/3D Pb-Sn alloyed perovskite solar cells. Nano Energy 76, 104999 (2020).

34. Zhang J, Qin JJ, Wu T, Hu B. Doping induced orbit-orbit interaction between excitons while enhancing photovoltaic performance in tin perovskite solar cells. J Phys Chem Lett 11, 6996-7001 (2020).

35. Bai YJ, Qin JJ, Shi L, Zhang J, Wang MS et al. Amplified spontaneous emission realized by cogrowing large/small grains with self-passivating defects and aligning transition dipoles. Adv Opt Mater 7, 1900345 (2019).

36. Qin JJ, Zhang J, Bai YJ, Ma SB, Wang MS et al. Enabling selfpassivation by attaching small grains on surfaces of large grains toward high-performance perovskite LEDs. iScience 19, 378-387 (2019).

37. Tiguntseva E, Koshelev K, Furasova A, Tonkaev P, Mikhailovskii $V$ et al. Room-temperature lasing from Mie-resonant nonplasmonic nanoparticles. ACS Nano 14, 8149-8156 (2020).

38. Makarov S, Furasova A, Tiguntseva E, Hemmetter A, Berestennikov $\mathrm{A}$ et al. Halide-perovskite resonant nanophotonics. Adv Opt Mater 7, 1800784 (2019).

39. deQuilettes DW, Frohna K, Emin D, Kirchartz T, Bulovic V et al. Charge-carrier recombination in halide perovskites. Chem Rev 119, 11007-11019 (2019).

40. Berestennikov AS, Voroshilov PM, Makarov SV, Kivshar YS. Active meta-optics and nanophotonics with halide perovskites. Appl Phys Rev 6, 031307 (2019).

41. Makarov SV, Milichko V, Ushakova EV, Omelyanovich M, Pasaran AC et al. Multifold emission enhancement in nanoimprinted hybrid perovskite metasurfaces. ACS Photonics 4, 728-735
(2017).

42. Li MX, He L, Xu HX, Shao M, Tisdale $\mathrm{J}$ et al. Interaction between optically-generated charge-transfer states and magnetized charge-transfer states toward magneto-electric coupling. J Phys Chem Lett 6, 4319-4325 (2015).

43. Perry CH, Lu F, Namavar F, Kalkhoran NM, Soref RA. Photoluminescence spectra from porous silicon (111) microstructures: temperature and magnetic-field effects. Appl Phys Lett 60, 3117-3119 (1992).

44. Hsiao YC, Wu T, Li MX, Hu B. Magneto-optical studies on spindependent charge recombination and dissociation in perovskite solar cells. Adv Mater 27, 2899-2906 (2015).

45. Zhang J, Wu T, Duan JS, Ahmadi M, Jiang FY et al. Exploring spin-orbital coupling effects on photovoltaic actions in $\mathrm{Sn}$ and $\mathrm{Pb}$ based perovskite solar cells. Nano Energy 38, 297-303 (2017).

46. Jones MAG, Morton JJL, Taylor RA, Ardavan A, Briggs GAD. $\mathrm{PL}$, magneto-PL and PLE of the trimetallic nitride template fullerene $\mathrm{Er}_{3} \mathrm{~N} @ \mathrm{C}_{80}$. Phys Status Solidi (B) 243, 3037-3041 (2006).

47. Pushkarev AP, Korolev VI, Markina DI, Komissarenko FE, Naujokaitis $\mathrm{A}$ et al. A few-minute synthesis of $\mathrm{CsPbBr}_{3}$ nanolasers with a high quality factor by spraying at ambient conditions. ACS Appl Mater Interfaces 11, 1040-1048 (2019).

\section{Acknowledgements}

This work was supported by the 973 Program and China National Key Basic Research Program (2016YFA0301100, 2016YFA0302000, and 2018YFA0306201), National Science Foundation of China (11774063 and 11727811), Science and Technology Commission of Shanghai Municipality (19XD1434600, 2019SHZDZX01 and 19DZ2253000), Air Force Office of Scientific Research (AFOSR) under the grant number FA 9550-15-1-0064, AOARD (FA2386-15-1-4104) and National Science Foundation (NSF1911659).

\section{Author contributions}

T. Y. Shen, J. J. Qin and Y. J. Bai contributed equally to this work. T. Y. Shen and Y. J. Bai performed all luminescence measurements and collected the scanning electron microscope and optical microscope images. J. J. Qin synthesized the perovskite (MAPbBr3) films. B. Y. Hou, J. Zi and L. Shi. supervised the work. T. Y. Shen, J. J. Qin, B. Hu. and L. Shi wrote the manuscript. All authors discussed the results and commented on the manuscript. The authors thank Ideaoptics Inc. for the support on the angle-resolved spectral characterizations and absorption measurements.

\section{Competing interests}

The authors declare no competing financial interests.

\section{Supplementary information}

Supplementary information for this paper is available at https://doi.org/10.29026/oea.2022.200051 